\title{
Fusobacterium nucleatum tumor DNA levels are associated with survival in colorectal cancer patients
}

\author{
Andrew T. Kunzmann ${ }^{1}$ • Marcela Alcântara Proença ${ }^{2}$ • Haydee WT Jordao ${ }^{1}$ • Katerina Jiraskova ${ }^{3,4}$. \\ Michaela Schneiderova ${ }^{5}$ - Miroslav Levy ${ }^{6}$ - Václav Liska ${ }^{7}$ - Tomas Buchler ${ }^{8}$ • Ludmila Vodickova ${ }^{3,4,7}$. \\ Veronika Vymetalkova $^{3,4,7}$ • Ana Elizabete Silva ${ }^{2}$ - Pavel Vodicka ${ }^{3,4,7}$ • David J. Hughes ${ }^{9}$ (D)
}

Received: 16 May 2019 / Accepted: 27 June 2019 / Published online: 31 July 2019

(C) The Author(s) 2019

\begin{abstract}
There is increasing evidence indicating a role for Fusobacterium nucleatum (F. nucleatum) in colorectal cancer (CRC) development and prognosis. This study evaluated $F$. nucleatum as a prognostic biomarker, by assessing its association with post-diagnosis survival from CRC. From September 2008 to April 2012 CRC patients $(n=190)$ were recruited from three hospitals within the Czech Republic. F. nucleatum DNA copies were measured in adjacent non-malignant and colorectal tumor tissues using quantitative real-time PCR. Cox Proportional Hazards (HR) models were applied to evaluate the association between $F$. nucleatum DNA and overall survival, adjusting for key confounders. Risk prediction modeling was conducted to evaluate the ability to predict survival based on F. nucleatum status. High, compared with low, levels of $F$. nucleatum in colorectal tumor tissues were associated with poorer overall survival (adjusted HR 1.68,95\% CI 1.02-2.77), which was slightly attenuated after additional adjustment for microsatellite instability status. However, inclusion of $F$. nucleatum in risk prediction models did not improve the ability to identify patients who died beyond known prognostic factors such as disease pathology staging. Although the increased presence of F. nucleatum was associated with poorer prognosis in CRC patients, this may have limited clinical relevance as a prognostic biomarker.
\end{abstract}

Keywords Colorectal neoplasm · Colorectal cancer $\cdot$ Fusobacterium nucleatum $\cdot$ Bacterial infection · Disease survival

\section{Introduction}

Accumulating evidence indicates a potential association of microbiome dysbiosis with colorectal cancer (CRC) development and prognosis $[1,2]$.

Electronic supplementary material The online version of this article (https://doi.org/10.1007/s10096-019-03649-1) contains supplementary material, which is available to authorized users.

Andrew T. Kunzmann

A.Kunzmann@qub.ac.uk

David J. Hughes

david.hughes@ucd.ie

1 Centre for Public Health, Queen's University Belfast, Belfast, Northern Ireland

2 Department of Biology, São Paulo State University, UNESP, São José do Rio Preto, SP, Brazil

3 Institute of Biology and Medical Genetics, First Faculty of Medicine, Charles University, Prague, Czech Republic

4 Department of Molecular Biology of Cancer, Institute of Experimental Medicine of the Czech Academy of Sciences, Prague, Czech Republic
Fusobacterium nucleatum (F. nucleatum) is an anaerobic, gram-negative commensal pathogen that is associated with several human diseases, especially those related to the oral and intestinal tract $[3,4]$. Numerous studies have shown higher abundance of $F$. nucleatum DNA or RNA in

Department of Surgery, General University Hospital in Prague, Prague, Czech Republic

6 Department of Surgery, First Faculty of Medicine, Charles University and Thomayer Hospital, Prague, Czech Republic

7 Biomedical Centre, Faculty of Medicine in Pilsen, Charles University, Pilsen, Czech Republic

8 Department of Oncology, First Faculty of Medicine, Charles University and Thomayer Hospital, Prague, Czech Republic

9 Cancer Biology and Therapeutics Group, School of Biomolecular and Biomedical Science, UCD Conway Institute, University College Dublin, Dublin D04 V1W8, Ireland 
CRC tumors versus surrounding non-malignant mucosa, or in stool sample DNA from CRC patients compared to controls [5-12] (reviewed in [13]). These findings are consistent with in vitro and animal model evidence and human studies indicating an etiological role of F. nucleatum in colorectal carcinogenesis through promotion of an immunocompromised proinflammatory microenvironment favorable to tumor initiation and progression [5, 14-17]. Alternatively, growth of $F$. nucleatum may simply reflect an opportunistic invader capable of surviving the harsh hypoxic conditions of developing tumors [8].

Regarding CRC pathology and clinical characteristics, high levels of $F$. nucleatum in stool and tumor tissue have been associated with right-sided tumors, higher stage, molecular subtypes, and worse clinical outcome (reviewed in [7, 18-20]). Several studies in diverse geographical settings, following the initial observation by Flanagan et al. (2014) [11], suggest that $F$. nucleatum may affect clinical outcome for CRC patients. These studies observed that higher tumor $F$. nucleatum levels were associated with poorer overall survival (OS) in CRC patients from the Czech Republic, China, Japan [11, 21-23], and metastatic CRC in South Korea [24]. The Chinese study from Wei et al. also reported an association with lower disease-free survival (DFS) [22]. Although a US study reported no association between high tumor $F$. nucleatum levels and OS in a large cohort of CRC patients, they did observe an association with shorter CRC-specific survival independent of clinical, pathological, and tumor molecular characteristics [25].

However, given the limited number of published studies to date, there is a need to further examine the relationship between F. nucleatum and CRC prognosis, and to what extent measurement of $F$. nucleatum DNA may serve as a prognostic biomarker. The aims of this study were to assess the association between F. nucleatum DNA levels from tumor and adjacent nonmalignant tissue with survival outcomes in a much larger and separate cohort of 190 Czech patients with CRC to our initial study suggesting a link with poorer overall patient survival [11].

\section{Materials and methods}

\section{Study population}

This cohort study included 190 adult patients diagnosed with histologically confirmed CRC. All subjects were recruited from September 2008 to April 2012 from one of three hospitals in the Czech Republic (General University Hospital, Thomayer Hospital in Prague and Biomedical Centre, Faculty of Medicine in Pilsen). Table 1 lists the participant characteristics.

\section{Data collection and follow-up}

A structured questionnaire assessed patients' personal characteristics (either self-reported or through interviews with their doctors), including date of birth, sex, lifestyle habits, body mass index (BMI), diabetes, and personal/family history of cancer. Clinicians recorded information about tumor location, tumor-node-metastasis (TNM) stage system, degree of tumor differentiation, and adjuvant chemotherapy treatment details. Patients with Lynch syndrome, polyposis syndromes, inflammatory bowel disease, or incomplete information on baseline characteristics were excluded. Follow-up information about distant metastasis, relapse, and date of death was also recorded and all registered patients were followed from surgery to death or to the end of the study (September 2018).

\section{DNA extraction}

After surgical resection, colorectal tumor and adjacent nonmalignant tissue samples were freshly frozen and conserved in either liquid nitrogen or RNA later and stored at $-80{ }^{\circ} \mathrm{C}$. DNA was extracted from colorectal and adjacent tissues using the DNeasy Blood and Tissue Kit and by following the manufacturer's instructions (Qiagen, Courtaboeuf, France). The DNA concentration was determined using the Quant-IT dsDNA BR Assay Kit (Life Technologies Czech Republic s.r.o., Prague, Czech Republic) and an Infinite M200 fluorescence reader (Tecan Group Ltd., Männedorf, Austria). Microsatellite instability (MSI) status was determined in tumor tissue and non-malignant adjacent mucosa using a pentaplex PCR assay of 5 mononucleotide repeat markers (BAT25, BAT26, NR21, NR24, NR27) with fluorescently labeled primers and standard PCR chemistry [26]. Fragment analysis was performer on ABI 3130 (Applied Biosystems, Foster City, CA, USA). The final comparison between tumor and non-tumor DNA short tandem repeat profiles was performed with GeneMapper v4.1 software (Applied Biosystems, Foster City, CA, USA). A tumor specimen was classified as MSI-high when 2 or more loci were unstable [26]. Mutation analysis for $B R A F, K R A S, N R A S$, and $P I K 3 C A$ was performed using a Randox mutation biochip array (Randox Laboratories Ltd., Crumlin, Northern Ireland) according to the manufacturer's instructions, as previously described [27].

\section{Quantitative real-time polymerase chain reaction}

Quantitative real-time polymerase chain reaction (qPCR) measured the number of copies of $F$. nucleatum DNA (nus $G$ gene), and a control human prostaglandin transporter ( $P G T)$ gene, in the colorectal tumor and non-malignant adjacent tissue using an Applied Biosystems 7500 Real-Time PCR System Levels, as previously detailed [11]. qPCR assays for 
Table 1 Participant characteristics in a cohort of 190 colorectal cancer patients based on F. nucleatum DNA status in colorectal cancer tissue

\begin{tabular}{|c|c|c|c|c|c|}
\hline & \multicolumn{2}{|c|}{ Low tumor $F$. nucleatum ${ }^{1}$} & \multicolumn{2}{|c|}{ High tumor F. nucleatum ${ }^{2}$} & \multirow[t]{2}{*}{$P$ value } \\
\hline & No. & $\%$ & No. & $\%$ & \\
\hline Total & 129 & $(68.2)$ & 61 & $(31.8)$ & \\
\hline \multicolumn{6}{|l|}{ Age at surgery } \\
\hline Under 60 & 22 & $(17.1)$ & 9 & $(14.8)$ & \multirow{4}{*}{0.95} \\
\hline $60-<70$ & 51 & $(39.5)$ & 23 & $(37.7)$ & \\
\hline $70<80$ & 36 & $(27.9)$ & 19 & $(31.1)$ & \\
\hline $80+$ & 20 & $(15.5)$ & 10 & (16.4) & \\
\hline \multicolumn{6}{|l|}{ Sex } \\
\hline Female & 40 & (31) & 20 & $(32.8)$ & \multirow{2}{*}{0.81} \\
\hline Male & 89 & (69) & 41 & $(67.2)$ & \\
\hline \multicolumn{6}{|l|}{ Tumor stage } \\
\hline I & 25 & (19.4) & 9 & $(14.8)$ & \multirow[t]{5}{*}{0.238} \\
\hline II & 36 & (27.9) & 23 & $(37.7)$ & \\
\hline III & 44 & $(34.1)$ & 14 & $(23)$ & \\
\hline IV & 22 & $(17.1)$ & 14 & (23) & \\
\hline Unknown & 2 & $(1.6)$ & 1 & (1.6) & \\
\hline \multicolumn{6}{|l|}{ Tumor location } \\
\hline Proximal colon & 39 & $(30.2)$ & 22 & $(36.1)$ & \multirow[t]{3}{*}{0.58} \\
\hline Distal colon & 38 & $(29.5)$ & 14 & $(23)$ & \\
\hline Rectal & 52 & $(40.3)$ & 25 & (41) & \\
\hline \multicolumn{6}{|c|}{ Neo/adjuvant treatment ${ }^{3}$} \\
\hline No & 89 & (69) & 34 & $(55.7)$ & \multirow[t]{2}{*}{0.07} \\
\hline Yes & 40 & (31) & 27 & $(44.3)$ & \\
\hline \multicolumn{6}{|l|}{ Smoking history } \\
\hline Never & 48 & $(43.6)$ & 24 & $(45.3)$ & \multirow[t]{3}{*}{0.84} \\
\hline Ever & 62 & $(56.4)$ & 29 & $(54.7)$ & \\
\hline Missing & 19 & $(14.5)$ & 8 & $(13.1)$ & \\
\hline \multicolumn{6}{|l|}{ Body Mass Index } \\
\hline $18.5-<25$ & 49 & $(38)$ & 18 & $(29.5)$ & \multirow[t]{4}{*}{0.61} \\
\hline $25-<30$ & 39 & $(30.2)$ & 21 & $(34.4)$ & \\
\hline $30+$ & 16 & $(12.4)$ & 7 & $(11.5)$ & \\
\hline Missing & 25 & (19.4) & 15 & $(24.6)$ & \\
\hline \multicolumn{6}{|c|}{ F. nucleatum DNA in non-malignant, adjacent tissue } \\
\hline Low $^{1}$ & 117 & $(90.7)$ & 36 & $(59.0)$ & \\
\hline $\mathrm{High}^{2}$ & 10 & $(7.8)$ & 24 & $(39.3)$ & $<0.001$ \\
\hline Missing & 2 & $(1.6)$ & 1 & $(1.6)$ & \\
\hline Microsatellite inst & & & & & \\
\hline MSS/MSI-L & 105 & $(81.4)$ & 40 & $(65.6)$ & \\
\hline MSI-H & 3 & $(2.3)$ & 10 & (16.4) & $<0.001$ \\
\hline Missing & 21 & (16.3) & 11 & (18.0) & \\
\hline$K R A S$ status & & & & & \\
\hline Wild type & 57 & $(44.2)$ & 18 & $(29.5)$ & \\
\hline Mutated & 30 & (23.3) & 17 & (27.9) & 0.15 \\
\hline Missing & 42 & (32.6) & 26 & (42.6) & \\
\hline$N R A S$ status & & & & & \\
\hline Wild type & 80 & $(62.0)$ & 34 & $(55.7)$ & \\
\hline Mutated & 7 & $(5.4)$ & 1 & $(1.6)$ & 0.30 \\
\hline Missing & 42 & $(32.6)$ & 26 & $(42.6)$ & \\
\hline$B R A F$ status & & & & & \\
\hline Wild type & 81 & $(62.8)$ & 31 & $(50.8)$ & \\
\hline Mutated & 6 & $(4.7)$ & 4 & $(6.6)$ & 0.41 \\
\hline Missing & 42 & (32.6) & 26 & $(42.6)$ & \\
\hline PIK3CA status & & & & & \\
\hline Wild type & 78 & $(60.5)$ & 31 & $(50.8)$ & \\
\hline Mutated & 9 & $(7.0)$ & 4 & $(6.6)$ & 0.86 \\
\hline Missing & 42 & (32.6) & 26 & $(42.6)$ & \\
\hline
\end{tabular}

${ }^{1}$ No F. nucleatum DNA detected within 42 PCR cycles or $F$. nucleatum $\left(2^{-\Delta \mathrm{CT}}\right)$ below 0.0008 (i.e., the median among individuals with tumor F. nucleatum DNA detected in 42 PCR cycles)

${ }^{2}$ F. nucleatum $\left(2^{-\Delta \mathrm{CT}}\right)$ above 0.0008

${ }^{3}$ Neo-adjuvant or adjuvant radiotherapy or chemotherapy within 6 months of surgery 
F. nucleatum were performed in duplicate for each sample, and a cutoff level of 42 replication cycles (cycle threshold; $\mathrm{CT}$ ) for both duplicate reactions was used to determine presence of F. nucleatum DNA. Bacterial abundance was calculated in both colorectal tumor tissue and adjacent tissue by $2^{-\Delta \mathrm{CT}}$, where $\Delta \mathrm{CT}$ is the difference in the $\mathrm{CT}$ number for $F$. nucleatum and the $P G T$ reference gene assay. F. nucleatum abundance (in individuals with the bacterium detected) was divided into low and high groups based on values below or above the median value, respectively.

\section{Statistical analysis}

All statistical analyses were performed using Stata version 14 (StataCorp, College Station, TX, USA).

The characteristics of patients based on the prevalence of F. nucleatum were compared using chi-squared tests. KaplanMeier curves and Cox Proportional Hazards ratio (HR) models with $95 \%$ confidence intervals $(95 \% \mathrm{CI})$ were applied to evaluate the association between $F$. nucleatum in colorectal tissue and overall survival. Both univariate and multivariate analyses were conducted, adjusted for important confounders such as age, sex, tumor stage, and chemotherapy or radiotherapy.

Sensitivity analyses assessed whether the association remained when conducting complete case analysis and separately when excluding deaths within the first 6 months. Further models included additional potential confounders associated with a $10 \%$ change in coefficient between F. nucleatum and OS, when added individually to the main multivariate model. The factors tested for inclusion were molecular characteristics (MSI status, mutations for $B R A F$, $K R A S, N R A S$, and PIK3CA) and lifestyle factors (including BMI and smoking history), tumor location, and the presence of F. nucleatum in adjacent non-malignant tissue. MSI status and $F$. nucleatum in adjacent non-malignant tissue were selected as additional covariates. Continuous and tertile category analyses were also conducted for assessing both F. nucleatum tumor abundance $\left(2^{-\Delta \mathrm{CT}}\right)$ and bacterial quantification in disease tissue over adjacent-matched colorectal tissue $\left(2^{-\Delta \Delta \mathrm{CT}}\right)$. The three categories included "no/low," where values of $F$. nucleatum were not detectable or if the quantification was in the lowest tertile group; "moderate" if bacterial abundance was in the middle tertile category; or "high" if F. nucleatum quantification was in the highest tertile rank.

In subgroup analyses, likelihood ratio tests assessed whether the association differed by age, sex, tumor stage, tumor location, chemotherapy or radiotherapy, smoking history, BMI, or by molecular characteristics (including status of MSI, BRAF, KRAS, NRAS, and PIK3CA).

The use of $F$. nucleatum load as a prognostic marker was examined by assessing the area under the receiver operating characteristics (ROC) curve of existing prognostic markers (tumor stage, age, sex, cancer treatment, and MSI status) when including and excluding tumor F. nucleatum status (high versus low).

\section{Results}

This study included 190 CRC patients who were followed up for a median of 2.34 years (range 0.01-6.77), during which time 71 patients died.

As observed in several studies [13], F. nucleatum was significantly more abundant in the tumor tissue compared to the matched surrounding mucosa $(p=0.002)$. Table 1 outlines the participant characteristics according to the abundance of F. nucleatum in CRC tumor tissue. One third of CRC patients had high $F$. nucleatum abundance in their tumor tissue. Patients with low and high abundance of $F$. nucleatum were similar in terms of age, sex, tumor stage and location, neoadjuvant treatment receipt, smoking history, and BMI. Patients with high tumor $F$. nucleatum were more likely to have MSI tumors than patients with low tumor $F$. nucleatum and tended to have a higher abundance of $F$. nucleatum in the adjacent-matched non-malignant tissue.

The association between tumor F. nucleatum status and overall survival is displayed in Table 2 and Fig. 1. There was no association with survival in the unadjusted analyses. However, high $F$. nucleatum was associated with poorer OS compared to low levels of the bacterium in colorectal tissue in fully adjusted models (HR 1.68, 95\% CI 1.02-2.77, $p=0.04$ ). Further adjustment for MSI status and $F$. nucleatum abundance in adjacent normal tissue did not markedly change this result, although it did lose statistical significance (HR 1.80, 95\% CI 0.97-3.28, $p=0.06$; see Supplementary Table 1). Additional factors considered in the adjustment model, comprising tumor colorectal subsite location, smoking history, BMI, and mutations of KRAS, NRAS, BRAF, and PIK3CA, did not meet the $10 \%$ change in coefficient criteria for confounder selection.

Sensitivity analyses for whether the association remained when conducting complete case analysis or after excluding deaths within the first 6 months did not substantially change the HR estimates, although the latter lost significance (HR 1.71, 95\% CI 0.94-3.10; Supplementary Table 1). No associations between tumor $F$. nucleatum status and OS were observed when analyses were stratified by CRC molecular subtypes (including $B R A F, K R A S, N R A S$, and PIK3CA mutation status) (Supplementary Table 1). The association between F. nucleatum and survival was significant in patients aged over 70 years old (HR 2.23, 95\% CI 1.15-4.35, $p=0.02$ ), in patients with left-sided tumors (HR 2.34, 95\% CI 1.25-4.37, $p=$ 0.008 ), and in those patients not receiving chemotherapy and/ or radiotherapy (HR 1.87, 95\% CI 1.02-3.45, $p=0.04$ ). However, formal tests for statistical interaction were not 
Table 2 Cox Proportional Hazards models for the association between Fusobacterium nucleatum DNA in colorectal cancer tissue and overall survival

\begin{tabular}{|c|c|c|c|c|c|c|}
\hline \multirow[t]{2}{*}{ F. nucleatum DNA in colorectal cancer tissue $\left(2^{-\Delta \mathrm{CT}}\right)$} & \multirow[t]{2}{*}{ Person-years } & \multirow[t]{2}{*}{ No. of events } & \multicolumn{2}{|l|}{ Unadjusted } & \multicolumn{2}{|l|}{ Adjusted $^{1}$} \\
\hline & & & HR $(95 \%$ CI $)$ & $p$ value & HR (95\% CI) & $p$ value \\
\hline Low/no & 376.2 & 44 & 1.00 (referent) & - & 1.00 (referent) & - \\
\hline High & 156.0 & 27 & $1.37(0.85-2.22)$ & 0.20 & $1.68(1.02-2.77)$ & 0.04 \\
\hline
\end{tabular}

${ }^{1}$ Adjusted for age $(<60,60<70,70<80,80+)$, sex (men, women), tumor stage (I, II, III, IV, missing), and chemotherapy or radiotherapy within 6 months (no, yes)

significant. Other clinical factors such as smoking status did not significantly modify the associations observed between F. nucleatum and OS (Supplementary Table 1).

Considering bacterial presence in the surrounding nonmalignant mucosal tissue, a multivariate analysis using relative quantification of $F$. nucleatum (from the mucosal and tumor levels) showed no association with OS (HR 1.04, 95\% CI 0.62-1.76; Supplementary Table 1). However, tumor levels adjusted for the corresponding $F$. nucleatum levels in the surrounding mucosal tissue (and the main confounders) were associated with poorer OS (HR 1.94, 95\% CI 1.093.46; Supplementary Table 1).

Assessing F. nucleatum levels in a continuous model provided null results (HR 1.00, 95\% CI 0.99-1.00). We were underpowered to conduct a restricted cubic spline analysis to explore the curve for the continuous model. However, although they were not significant, categorical analyses of no/ low, moderate, or high bacterial levels interestingly suggest that this is largely due to a non-linear relationship where CRC cases with moderate $F$. nucleatum have possibly better or equivalent survival than those with no/low $F$. nucleatum in their tumor tissues (see Supplementary Table 2). Survival then

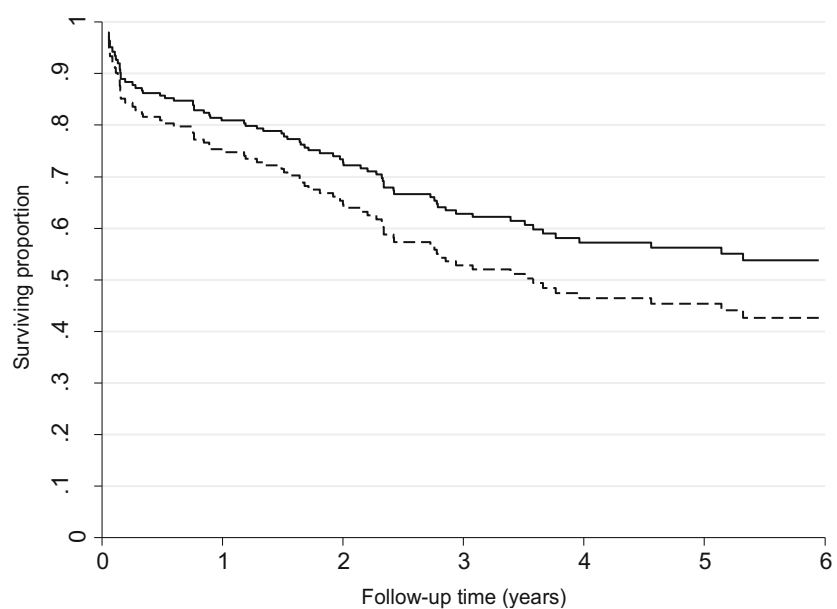

Fig. 1 Kaplan-Meier curve showing the overall survival of colorectal cancer patients by the amount of $F$. nucleatum DNA in colorectal tumor tissue $\left(2^{-\Delta \mathrm{CT}}\right)$; continuous line indicates low tumor $F$. nucleatum; broken line indicates high tumor $F$. nucleatum. In the adjusted models, high F. nucleatum was associated with poorer OS compared to lower levels (HR $1.68,95 \%$ CI $1.02-2.77, p=0.04$ ) decreases with higher bacterial load, as also indicated by the Kaplan-Meier survival curves in our prior report from 2014 (see figure 3, Flanagan et al., 2014 [11]). Alternatively, this may simply reflect lower power for these tertile groupings. Repeating these categorical analyses by the delta-delta $\mathrm{Ct}$ method used in Flanagan et al. [11] for comparing differences in bacterial levels between tumor and matched mucosal tissues, or by adjusting for $F$. nucleatum mucosal tissue levels, made no major differences to the results (Supplementary Table 2).

Finally, when the utility of $F$. nucleatum as a prognostic marker was assessed by ROC curves, there was no improvement in the area under the curve when added to traditional prognostic markers or data such as tumor stage, age, sex, treatment, and MSI status (Table 3).

\section{Discussion}

In this study, we found that high $F$. nucleatum DNA in colorectal tumor tissue is associated with shorter survival in a cohort of CRC patients from the Czech Republic. However, prognostic modeling revealed that $F$. nucleatum may not lead to notable improvements in our ability to predict patient prognosis beyond other known indicators, such as tumor stage.

The association of higher levels of $F$. nucleatum DNA with a shorter survival in CRC patients is concordant with previous reports [11, 21-25]. These include our initial study suggesting this link for the first time, although in a small, separate group of 32 Czech CRC patients giving imprecise HR estimates with wide confidence intervals [11]. The large study by Mima et al., of tissue from 1069 CRC cases within the Nurses' Health Study and the Health Professionals Follow-Up study, found that high $F$. nucleatum was more strongly associated with CRC-specific survival (HR 1.58, 95\% CI 1.04-2.39) than with OS (HR 1.08, 95\% CI $0.76-$ 1.52) [25]. Given the long duration of follow-up in this study (median of 10.7 years), analyses of OS understandably incorporated a high proportion of non-CRC deaths $(46 \%)$, likely underlying the null association with OS for F. nucleatum. Considering the other two of these reports which calculated HR values, then the strength of 
Table 3 Performance of prognostic models for five-year overall survival in colorectal cancer patients when including and excluding tumor $F$. nucleatum status

\begin{tabular}{lc}
\hline & AUROC (95\% CI) \\
\hline Tumor stage & $0.68(0.60-0.76)$ \\
Tumor stage + F. nucleatum & $0.69(0.61-0.77)$ \\
Age, sex, tumor stage & $0.78(0.72-0.85)$ \\
Age, sex, tumor stage + F. nucleatum & $0.78(0.71-0.85)$ \\
Age, sex, tumor stage, treatment & $0.78(0.71-0.84)$ \\
Age, sex, tumor stage, treatment + F. nucleatum & $0.78(0.71-0.84)$ \\
Age, sex, tumor stage, treatment, and MSI status & $0.83(0.77-0.89)$ \\
Age, sex, tumor stage, treatment, and MSI status $+F$. nucleatum & $0.83(0.77-0.89)$ \\
\hline
\end{tabular}

F. nucleatum, Fusobacterium nucleatum; AUROC, area under receiver operating characteristic curve, $95 \%$ CI, 95\% confidence intervals; $M S I$, microsatellite instability the association for higher $F$. nucleatum with worse OS seen in the current study (HR 1.68, 95\% CI 1.02-2.77) is similar to Chinese CRC patients (multivariate HR $1.99,95 \%$ CI 1.02-3.88) [22] and very close to the estimate for a South Korean metastatic CRC cohort (multivariate HR 1.69, 95\% CI 1.04-2.75) [24]. Therefore, the current study provides additional support that $F$. nucleatum is independently associated with poorer OS for CRC patients. Contrastingly, a recent and separate South Korean study reported no association of bacterial levels with survival for 593 stage II (high-risk) and stage III CRC patients whom had all received oxaliplatin-based adjuvant chemotherapy [28]. This aligns with our observation of no association between F. nucleatum and OS in patients who had received chemotherapy or radiotherapy. Furthermore, in subgroup analyses, these authors also reported that higher $F$. nucleatum showed a non-significant tendency toward worse prognosis in sigmoid colon and rectal cancer but was significantly associated with a more favorable DFS for non-sigmoid colon cancer patients that were also nonMSI-high (i.e., MSS/MSI-low). We observed that the association of higher bacterial levels with worse prognosis was more apparent in patients with left-sided tumors (thus including sigmoid and rectal cancers, as well as the splenic flexure and descending colon). However, this Korean study was likely seriously confounded by study design and methodology issues such as the lack of qPCR data on adjacent-matched tissues for each tumor, and that FFPE tissue-derived DNA was used to estimate the bacterial load resulting in a high number of failed PCRs for the PGT control (21\%) so that the results are possibly unreliable. Indeed, tissue fixation method and storage time has been shown to affect $F$. nucleatum qPCR positivity [24, 28].

The biological mechanism for the association between F. nucleatum and patient survival has not been fully elucidated. The bacterium has been shown to persist in cancer cells from metastatic CRC lesions and Fusobacterium-culture positive tumors resulted in more successful xenografts in mice, suggesting an association with more severe disease [29].
Human and functional studies provide evidence that F. nucleatum-mediated increased gut inflammation and chemoresistance, through immune signaling and autophagy activation, largely explains the poorer prognosis for CRC patients $[22,30]$. F. nucleatum may invade into tumor cells and exacerbate inflammatory and oncogenic responses via FadA adhesion to E-cadherin with beta-catenin pathway activation [5] and through Fap2 binding of the Gal-GalNAc polysaccharide overexpressed by CRC cells [15]. Additionally, Fap2 interaction with the TIGIT receptor expressed on natural killer cells may enhance immune evasion [17]. The bacterium is also associated with upregulation of several inflammatory cytokines through a postulated miRNA-mediated activation of TLR2/TLR4 [31] and activation of the JAK/STAT and MAPK/ERK pathways linked to CRC tumor progression [32]. Alternatively, as in the case for any role in initial colorectal carcinogenesis, $F$. nucleatum may not directly affect CRC disease severity, but simply thrive in the increasingly hostile tumor microenvironment of aggressive tumors that are likely to be associated with a poorer prognosis. In contrast to the suggested role of chemoresistance, in subgroup analyses, we only observed an association between higher $F$. nucleatum and OS in patients who did not receive chemotherapy or radiotherapy. However, our power was very limited to adequately evaluate this due to low numbers of patients with both high bacterial levels and relevant therapy data, and formal tests for statistical interaction were not significant. Further analyses by continuous or category (no/low, moderate, and high) levels of $F$. nucleatum possibly suggest that moderate amounts may be conversely associated with better or equal survival than for patients with no/low presence of the bacterium in their tumor tissue. Conceivably, this could be through triggering of an increased immune response that may combat tumor progression/recurrence until the bacterium reaches higher amounts that would compromise therapy and/or make the cancer more aggressive. Alternatively, this may again reflect a non-causative correlation between higher bacterial 
levels in more severe cancers. Although, considerable caution is required in assessing these results, as none were significant and there was lower power for these evaluations.

In analyses by CRC molecular subtypes, we found that the association between $F$. nucleatum and overall survival was no longer statistically significant after additional adjustment for MSI status and presence of the bacterium in adjacent non-malignant tissue. This may be attributable to low statistical power, as few individuals were MSI-high ( $n=13)$. Stratified analysis in patients with MSI-low/stable colorectal tumors, or by $B R A F, K R A S$, $N R A S$, and PIK3CA mutation status, also showed no evidence of an association between F. nucleatum and OS. Unfortunately, we had insufficient statistical power to conduct this analysis for MSI-high tumors. Nonetheless, a strong association between the amount of $F$. nucleatum in CRC tissue and MSI-high was observed in the study by Mima et al. [25]. Although MSI-high status is generally perceived as a favorable prognostic indicator, a large cohort study of Stage II/III colon cancer patients from Northern Ireland defined a subgroup of patients having MSI-high tumors with a high fibroinflammatory score that were associated with a 2.5-fold increased risk of death [33]. The fibroinflammatory score is indicative of tumor microenvironment features such as peritumoral diffuse lymphoid inflammation, Crohn's disease-like reaction, and tumor stromal proportions [33]. However, as we were unable to measure this directly with our limited sample size, it would be interesting for further studies to test whether such a group of MSI-high/ fibroinflammatory-high tumors correlate with increased F. nucleatum abundance and poorer outcomes. Additionally, a positive and negative correlation has been reported for MSS/ MSI-low and MSI-high CRCs, respectively, with higher F. nucleatum levels and a clinically favorable increased tumorinfiltrating lymphocyte (TIL) density [34]. Thus, the association of increased TILs with F. nucleatum load in non-MSI-high tumors may improve the disease outcomes for these patients [28]. Overall, it appears that high F. nucleatum abundance is contributing to an adverse inflammatory tumor microenvironment that is associated with poorer outcomes, although tumor location and molecular subtype may be important factors modifying the influence of $F$. nucleatum on patient outcomes.

Additionally, F. nucleatum did not appear to be a useful prognostic tool for predicting individuals who may have worse survival when added to current known prognostic factors such as tumor stage. Although, we did not have information on CRCspecific survival, which may be more strongly related to F. nucleatum, it is likely that most deaths in this cohort were attributed to CRC considering the relatively short follow-up time (median of 2.34 years). Further, larger cohort studies with information on causes of death could examine the utility of F. nucleatum measurement regarding CRC-specific survival prognosis, with adequately powered analyses to assess whether the prognostic role is greater in certain tumor subsites, molecular subtypes, or patient subgroups.
Our study had several notable strengths and limitations. Strengths include the use of DNA extracted from freshfrozen tissue, with no tissue fixation, so that we had $>99 \%$ successful qPCR assays (based on consistent amplification of the human $P G T$ reference gene in all tumor and matched mucosa samples), and that we assessed the prognostic utility of F. nucleatum DNA measurement in CRC patients. However, the survival analyses were constricted to OS so we were not able to assess CRC-specific mortality, the cohort size was limited for many of the subgroup analyses, and that qPCR may not measure $F$. nucleatum as accurately as other approaches, such as droplet digital PCR used by Yamaoka et al. [23]. However, qPCR is the most widely accepted and used method of $F$. nucleatum measurement in other studies.

In conclusion, our study adds to the observational evidence of an association between high abundance of $F$. nucleatum in colorectal tumors and poorer survival outcomes. Additionally, as antibiotic treatment of colon cancer xenografts in mice has been shown to decrease $F$. nucleatum levels and tumor growth [29], trials testing whether treatments aimed at reducing the tumor F. nucleatum lead to improved clinical outcomes for CRC patients may be warranted. Although, our study further indicated that the utility of $F$. nucleatum for predicting prognosis is limited, this needs to be further assessed in independent datasets.

Acknowledgments We wish to thank Helen G Coleman and Gerry McKenna of the Centre for Public Health, Queen's University Belfast, Northern Ireland, for their helpful suggestions and respective supervision of ATK and HJ.

Author contributions Conceptualization, DJH, and PV; data curation, DJH, PV, KJ, VV, and ATK; formal analysis, ATK, MAP, HJ, and DJH; funding acquisition, DJH, PV, and AES; investigation, DJH, MAP, AES, PV, KJ, MS, ML, LV, VL, TB, and VV; methodology, DJH, MAP, and ATK; project administration, DJH, KJ, VV, LV, and PV; resources, DJH, PV, MS, ML, VL, and TB; supervision, DJH, PV; writing-original draft, ATK, HJ, and DJH; writing-review and editing, DJH and PV.

Funding Funding for this study was provided by the Health Research Board of Ireland health research award (HRA-PHS/2015/1142) and a Science Foundation Ireland-Brazil International Strategic Cooperation Award (13/ISCA/2843) to DJH, MAP, and AES. Support for this study was also provided by the COST Action CA17118 supported by COST (European Cooperation in Science and Technology, www.cost.eu) to DJH, VV, and PV. The co-authors from the Czech Republic received support from the Ministry of Health of the Czech Republic (AZV 1527580A and AZV NV 18-03-00199).

\section{Compliance with ethical standards}

Conflict of interest The authors declare that they have no conflict of interest.

Ethical approval The study was approved by the Ethical Committees of the Thomayer Hospital in Prague, General University Hospital at the 1st Medical Faculty, Charles University in Prague and Teaching Hospital in Pilsen (Czech Republic). 
Informed consent All recruited patients were asked to carefully read and sign an informed consent and the present research was performed in accordance with the ethical standards of the 1964 Declaration of Helsinki and its later amendments. All samples were coded to protect patient anonymity.

Open Access This article is distributed under the terms of the Creative Commons Attribution 4.0 International License (http:// creativecommons.org/licenses/by/4.0/), which permits unrestricted use, distribution, and reproduction in any medium, provided you give appropriate credit to the original author(s) and the source, provide a link to the Creative Commons license, and indicate if changes were made.

\section{References}

1. Gao R, Gao Z, Huang L, Qin H (2017) Gut microbiota and colorectal cancer. Eur J Clin Microbiol Infect Dis 36:757-769. https:// doi.org/10.1007/s10096-016-2881-8

2. Tilg H, Adolph TE, Gerner RR, Moschen AR (2018) The intestinal microbiota in colorectal cancer. Cancer Cell 33:954-964. https:// doi.org/10.1016/j.ccell.2018.03.004

3. Han YW (2015) Fusobacterium nucleatum: a commensal-turned pathogen. Curr Opin Microbiol 23:141-147. https://doi.org/10. 1016/j.mib.2014.11.013

4. Signat B, Roques C, Poulet P, Duffaut D (2011) Fusobacterium nucleatum in periodontal health and disease. Curr Issues Mol Biol 13:25-36

5. Rubinstein MR, Wang X, Liu W, Hao Y, Cai G, Han YW (2013) Fusobacterium nucleatum promotes colorectal carcinogenesis by modulating E-cadherin/beta-catenin signaling via its FadA adhesin. Cell Host Microbe 14:195-206. https://doi.org/10.1016/j.chom. 2013.07.012

6. Castellarin M, Warren RL, Freeman JD, Dreolini L, Krzywinski M, Strauss J, Barnes R, Watson P, Allen-Vercoe E, Moore RA, Holt RA (2012) Fusobacterium nucleatum infection is prevalent in human colorectal carcinoma. Genome Res 22(2):299-306. https://doi.org/ 10.1101/gr.126516.111

7. Brennan CA, Garrett WS (2019) Fusobacterium nucleatum - symbiont, opportunist and oncobacterium. Nat Rev Microbiol 17(3): 156-166. https://doi.org/10.1038/s41579-018-0129-6

8. Amitay EL, Werner S, Vital M, Pieper DH, Höfler D, Gierse IJ, Butt J, Balavarca Y, Cuk K, Brenner H (2017) Fusobacterium and colorectal cancer: causal factor or passenger? Results from a large colorectal cancer screening study. Carcinogenesis 38(8):781-788. https://doi.org/10.1093/carcin/bgx053

9. Feng Q, Liang S, Jia H, Stadlmayr A, Tang L, Lan Z, Zhang D, Xia H, Xu X, Jie Z, Su L, Li X, Li X, Li J, Xiao L, Huber-Schönauer U, Niederseer D, Xu X, Al-Aama JY, Yang H, Wang J, Kristiansen K, Arumugam M, Tilg H, Datz C, Wang J (2015) Gut microbiome development along the colorectal adenoma-carcinoma sequence. Nat Commun 6:6528. https://doi.org/10.1038/ncomms7528

10. Yu J, Yu J, Feng Q, Wong SH, Zhang D, Liang QY, Qin Y, Tang L, Zhao H, Stenvang J, Li Y, Wang X, Xu X, Chen N, Wu WK, AlAama J, Nielsen HJ, Kiilerich P, Jensen BA, Yau TO, Lan Z, Jia H, Li J, Xiao L, Lam TY, Ng SC, Cheng AS, Wong VW, Chan FK, Xu X, Yang H, Madsen L, Datz C, Tilg H, Wang J, Brünner N, Kristiansen K, Arumugam M, Sung JJ, Wang J (2015) Metagenomic analysis of faecal microbiome as a tool towards targeted non-invasive biomarkers for colorectal cancer. Gut 66(1): 70-78. https://doi.org/10.1136/gutjnl-2015-309800

11. Flanagan L, Schmid J, Ebert M, Soucek P, Kunicka T, Liska V, Bruha J, Neary P, Dezeeuw N, Tommasino M, Jenab M, Prehn JH, Hughes DJ (2014) Fusobacterium nucleatum associates with stages of colorectal neoplasia development, colorectal cancer and disease outcome. Eur J Clin Microbiol Infect Dis 33(8):1381-1390. https://doi.org/10.1007/s10096-014-2081-3

12. Warren RL, Freeman DJ, Pleasance S, Watson P, Moore RA, Cochrane K, Allen-Vercoe E, Holt RA (2013) Co-occurrence of anaerobic bacteria in colorectal carcinomas. Microbiome 1(1):16. https://doi.org/10.1186/2049-2618-1-16

13. Hussan H, Clinton SK, Roberts K, Bailey MT (2017) Fusobacterium's link to colorectal neoplasia sequenced: a systematic review and future insights. World J Gastroenterol 23(48): 8626-8650. https://doi.org/10.3748/wjg.v23.i48.8626

14. Kostic AD, Chun E, Robertson L, Glickman JN, Gallini CA, Michaud M, Clancy TE, Chung DC, Lochhead P, Hold GL, ElOmar EM, Brenner D, Fuchs CS, Meyerson M, Garrett WS (2013) Fusobacterium nucleatum potentiates intestinal tumorigenesis and modulates the tumor-immune microenvironment. Cell Host Microbe 14(2):207-215. https://doi.org/10.1016/j.chom. 2013.07.007

15. Abed J, Emgård JE, Zamir G, Faroja M, Almogy G, Grenov A, Sol A, Naor R, Pikarsky E, Atlan KA, Mellul A, Chaushu S, Manson AL, Earl AM, Ou N, Brennan CA, Garrett WS, Bachrach G (2016) Fap2 mediates fusobacterium nucleatum colorectal adenocarcinoma enrichment by binding to tumor-expressed Gal-GalNAc. Cell Host Microbe 20(2):215-225. https://doi.org/10.1016/j.chom.2016. 07.006

16. Mima K, Sukawa Y, Nishihara R, Qian ZR, Yamauchi M, Inamura K, Kim SA, Masuda A, Nowak JA, Nosho K, Kostic AD, Giannakis M, Watanabe H, Bullman S, Milner DA, Harris CC, Giovannucci E, Garraway LA, Freeman GJ, Dranoff G, Chan AT, Garrett WS, Huttenhower C, Fuchs CS, Ogino S (2015) Fusobacterium nucleatum and $\mathrm{T}$ Cells in colorectal carcinoma. JAMA Oncol 1(5):653-661. https://doi.org/10.1001/jamaoncol. 2015.1377

17. Gur C, Ibrahim Y, Isaacson B, Yamin R, Abed J, Gamliel M, Enk J, Bar-On Y, Stanietsky-Kaynan N, Coppenhagen-Glazer S, Shussman N, Almogy G, Cuapio A, Hofer E, Mevorach D, Tabib A, Ortenberg R, Markel G, Miklić K, Jonjic S, Brennan CA, Garrett WS, Bachrach G, Mandelboim O (2015) Binding of the Fap2 protein of fusobacterium nucleatum to human inhibitory receptor TIGIT protects tumors from immune cell attack. Immunity 42(2): 344-355. https://doi.org/10.1016/j.immuni.2015.01.010

18. Kelly D, Yang L, Pei Z (2018) Gut microbiota, fusobacteria, and colorectal cancer. Diseases 6(4):E109. https://doi.org/10.3390/ diseases6040109

19. Zhang S, Cai S, Ma Y (2018) Association between fusobacterium nucleatum and colorectal cancer: progress and future directions. J Cancer 9(9):1652-1659. https://doi.org/10.7150/jca.24048

20. Shang FM, Liu HL (2018) Fusobacterium nucleatum and colorectal cancer: a review. World J Gastrointest Oncol 10(3):71-81. https:// doi.org/10.4251/wjgo.v10.i3.71

21. Sun Y, An QM, Tian XY, Wang ZL, Guan XY, Dong B, Zhao M, Hao CY (2016) Fusobacterium nucleatum infection is correlated with tumor metastasis and postoperative survival of colorectal cancer patients in China. Transl Cancer Res 5(5):579-588. https://doi. org/10.21037/tcr.2016.10.45

22. Wei Z, Cao S, Liu S, Yao Z, Sun T, Li Y, Li J, Zhang D, Zhou Y (2016) Could gut microbiota serve as prognostic biomarker associated with colorectal cancer patients' survival? A pilot study on relevant mechanism. Oncotarget 7(29):46158-46172. https://doi. org/10.18632/oncotarget.10064

23. Yamaoka Y, Suehiro Y, Hashimoto S, Hoshida T, Fujimoto M, Watanabe M, Imanaga D, Sakai K, Matsumoto T, Nishioka M, Takami T, Suzuki N, Hazama S, Nagano H, Sakaida I, Yamasaki $\mathrm{T}$ (2018) Fusobacterium nucleatum as a prognostic marker of colorectal cancer in a Japanese population. J Gastroenterol 53(4):517524. https://doi.org/10.1007/s00535-017-1382-6 
24. Lee DW, Han SW, Kang JK, Bae JM, Kim HP, Won JK, Jeong SY, Park KJ, Kang GH, Kim TY (2018) Association between fusobacterium nucleatum, pathway mutation, and patient prognosis in colorectal cancer. Ann Surg Oncol 25(11):3389-3395. https:// doi.org/10.1245/s10434-018-6681-5

25. Mima K, Nishihara R, Qian ZR, Cao Y, Sukawa Y, Nowak JA, Yang J, Dou R, Masugi Y, Song M, Kostic AD, Giannakis M, Bullman S, Milner DA, Baba H, Giovannucci EL, Garraway LA, Freeman GJ, Dranoff G, Garrett WS, Huttenhower C, Meyerson M, Meyerhardt JA, Chan AT, Fuchs CS, Ogino S (2016) Fusobacterium nucleatum in colorectal carcinoma tissue and patient prognosis. Gut 65(12):1973-1980. https://doi.org/10.1136/gutjnl2015-310101

26. Suraweera N, Duval A, Reperant M, Vaury C, Furlan D, Leroy K, Seruca R, Iacopetta B, Hamelin R (2002) Evaluation of tumor microsatellite instability using five quasimonomorphic mononucleotide repeats and pentaplex PCR. Gastroenterology 123(6):18041811

27. Lemstrova R, Brynychova V, Hughes DJ, Hlavac V, Dvorak P, Doherty JE, Murray HA, Crockard M, Oliverius M, Hlavsa J, Honsova E, Mazanec J, Kala Z, Lovecek M, Havlik R, Ehrmann J, Strouhal O, Soucek P, Melichar B, Mohelnikova-Duchonova B (2017) Dysregulation of KRAS signaling in pancreatic cancer is not associated with KRAS mutations and outcome. Oncol Lett 14(5): 5980-5988. https://doi.org/10.3892/ol.2017.694

28. Oh HJ, Kim JH, Bae JM, Kim HJ, Cho NY, Kang GH (2019) Prognostic impact of fusobacterium nucleatum depends on combined tumor location and microsatellite instability status in stage II/III colorectal cancers treated with adjuvant chemotherapy. J Pathol Transl Med 53(1):40-49. https://doi.org/10.4132/jptm. 2018.11.29

29. Bullman S, Pedamallu CS, Sicinska E, Clancy TE, Zhang X, Cai D, Neuberg D, Huang K, Guevara F, Nelson T, Chipashvili O, Hagan T, Walker M, Ramachandran A, Diosdado B, Serna G, Mulet N, Landolfi S, Ramon Y, Cajal S, Fasani R, Aguirre AJ, Ng K, Élez E, Ogino S, Tabernero J, Fuchs CS, Hahn WC, Nuciforo P, Meyerson M (2017) Analysis of Fusobacterium persistence and antibiotic response in colorectal cancer. Science 358(6369):1443-1448. https:// doi.org/10.1126/science.aal5240
30. Yu T, Guo F, Yu Y, Sun T, Ma D, Han J, Qian Y, Kryczek I, Sun D, Nagarsheth N, Chen Y, Chen H, Hong J, Zou W, Fang JY (2017) Fusobacterium nucleatum promotes chemoresistance to colorectal cancer by modulating autophagy. Cell 170(3):548-563.e16. https:// doi.org/10.1016/j.cell.2017.07.008

31. Proença MA, Biselli JM, Succi M, Severino FE, Berardinelli GN, Caetano A, Reis RM, Hughes DJ, Silva AE (2018) Relationship between Fusobacterium nucleatum, inflammatory mediators and microRNAs in colorectal carcinogenesis. World J Gastroenterol 24(47):5351-5365. https://doi.org/10.3748/wjg.v24.i47.5351

32. Yu YN, Yu TC, Zhao HJ, Sun TT, Chen HM, Chen HY, An HF, Weng YR, Yu J, Li M, Qin WX, Ma X, Shen N, Hong J, Fang JY (2015) Berberine may rescue Fusobacterium nucleatum-induced colorectal tumorigenesis by modulating the tumor microenvironment. Oncotarget 6(31):32013-32026. https://doi.org/10.18632/ oncotarget.5166

33. Hynes SO, Coleman HG, Kelly PJ, Irwin S, O'Neill RF, Gray RT, McGready C, Dunne PD, McQuaid S, James JA, Salto-Tellez M, Loughrey MB (2017) Back to the future: routine morphological assessment of the tumour microenvironment is prognostic in stage II/III colon cancer in a large population-based study. Histopathology 71(1):12-26. https://doi.org/10.1111/his.13181

34. Hamada T, Zhang X, Mima K, Bullman S, Sukawa Y, Nowak JA, Kosumi K, Masugi Y, Twombly TS, Cao Y, Song M, Liu L, da Silva A, Shi Y, Gu M, Li W, Koh H, Nosho K, Inamura K, Keum N, Wu K, Meyerhardt JA, Kostic AD, Huttenhower C, Garrett WS, Meyerson M, Giovannucci EL, Chan AT, Fuchs CS, Nishihara R, Giannakis M, Ogino S (2018) Fusobacterium nucleatum in colorectal cancer relates to immune response differentially by tumor microsatellite instability status. Cancer Immunol Res 6(11):13271336. https://doi.org/10.1158/2326-6066.CIR-18-0174

Publisher's note Springer Nature remains neutral with regard to jurisdictional claims in published maps and institutional affiliations. 